\title{
CONSTRUCCIÓN DE SIGNIFICADOS EN UN DISCURSO OFICIAL (SAN VITO, 1982)
}

\section{SIGNIFICANCE CONSTRUCTION IN AN OFFICIAL SPEECH (SAN VITO, 1982)}

\section{Annamaria Rimolo Bariatti*}

\section{RESUMEN}

El propósito de este artículo es indagar la posibilidad de una construcción de significados en el discurso enunciado el 28 de febrero de 1982, por parte del Ing. Agr. Claudio Antonio Volio Guardia, ex Ministro de Agricultura (1949-1953), a la luz del análisis del discurso según Teun A. van Dijk. Para ello se explora sus conceptos más relevantes (acción, contexto, poder e ideología) para establecer la conexión entre discurso y sociedad en nuestra modernidad.

\begin{abstract}
PALABRAS CLAVE: TEUN A. VAN DIJK * DISCURSO * ANÁLISIS * ACCIÓN * CONTEXTO * PODER * IDEOLOGÍAS
\end{abstract}

\section{ABSTRACT}

The aim of this essay is to explore the possibility of significance construction in the speech given by the Agronomical Engineer Claudio Antonio Volio Guardia, former Costa Rican Minister of Agriculture (1949-1953) on February 28, 1982 in accordance to the discourse analysis after Teun A. van Dijk. In order to achieve this goal we explored his most relevant concepts (action, context, power and ideology) trying to establish the connection between discourse and society within our modernity.

KEYWORDS: TEUN A VAN DIJK * SPEECHES * ANALYSIS * ACTION * CONTEXT * POWER * IDEOLOGIES

Escuela de Lenguas Modernas de la Universidad de Costa Rica (UCR). 


\section{INTRODUCCIÓN}

Para Teun A. van Dijk, los usuarios del lenguaje al utilizar habla $y$ texto, llevan a cabo acciones de índole política o social (2008). Desde esta perspectiva, las nociones de "acción", "contexto", "poder" e "ideología" se revisten de gran importancia y se ocupa de estas a lo largo de su obra. El objetivo del presente artículo es analizar el discurso político, enunciado el 28 de setiembre de 1982, por parte del ex Ministro de Agricultura (1949-1953) Ing. Agr. Claudio Antonio Volio Guardia en conmemoración de los 30 años de San Vito de Java, siguiendo los postulados básicos del análisis del discurso como interacción en la sociedad según Teun A. van Dijk (Ibid).

El pensamiento de Teun A. van Dijk en relación a los estudios sobre análisis del discurso, es vasto $y$ largo ${ }^{1}$. Sugiere que en todos los niveles del discurso se pueden encontrar "huellas del contexto" (van Dijk, 2012) que indican las características de los usuarios del lenguaje (sexo, clase, edad, origen, posición, pertenencia grupal y prejuicios étnicos). Aboga por la hipótesis de que las élites desempeñan un papel decisivo en la reproducción de categorías ideológicas (van Dijk, 2003). Asimismo, en el discurso del ex ministro se puede entrever las políticas eugenésicas del discurso institucional costarricense con la fundación de San Vito de Java y la colonización de 10000 hectáreas de selva en Coto Brus, por un grupo de inmigrantes italianos bajo el comando de don Vito Sansonetti.

En el primer apartado de este artículo, se realiza la narración de la fundación de San Vito de Java, su reseña histórica y su relación con el ex ministro de agricultura (1949-1953), Ing. Agr. Claudio Antonio Volio Guardia. En el segundo apartado, se analiza el discurso político del ex ministro, siguiendo los presupuestos conceptuales fundamentales: acción, contexto, poder e ideología. Aquí se pretende analizar la construcción de significados en el discurso enunciado en 1982 por Volio Guardia en conmemoración de los 30 años de San Vito de Java.

1 Consultar página del autor <http://www.discursos. org>

\section{SAN VITO DE JAVA Y EL DISCURSO INSTITUCIONAL COSTARRICENSE}

San Vito de Java se localiza, según el decreto ejecutivo nro. 10 del 31 de enero de 1952 (República de Costa Rica; 1952: 47-48), entre los ríos Negro y Sabalito, desde la confluencia de ambos ríos, aguas abajo, hasta llegar al Río Coto Brus. Se encuentra en la Zona Sur de Costa Rica, en la provincia de Puntarenas y es cabecera del cantón de Coto Brus. Dicho cantón, según la última estimación al 30 de junio de 2009, cuenta con una población de 47930 habitantes (INEC, 2009) y limita al norte con el cantón de Buenos Aires y Panamá, al este con Panamá, al sur y al oeste con los cantones de Golfito y Buenos Aires.

El surgimiento de esta comunidad se da en el inicio de la década de 1950, a partir de la colonización agrícola de la región por parte de varias familias italianas. Fue fundada por una empresa colonizadora privada con características propias (Bariatti, 2001) en el sentido de que los emigrantes italianos procedían de varias regiones de Italia, quienes se trasladaron a un mismo lugar (fenómeno demográfico único en las migraciones italianas hacia Costa Rica) y con un propósito compartido: colonizar un territorio inhóspito en aras de una mejor vida. Este fenómeno introdujo la categoría laboral de 'colono' entre los italianos residentes en Costa Rica. Además, fue la primera colonización agrícola extranjera exitosa en el país en contraposición a otros proyectos colonizadores italianos, como los fallidos intentos en la península de Uvita, en 1927 y en la zona de Moravia de Chirripó, a principios de 1952 (Hall, 1976; Weizmann, 1985; Sansonetti, Vito, 1995; Sansonetti, Ugo; 2000 y Bariatti, 2001).

El mencionado contrato estipulaba que el Gobierno de Costa Rica debía destinar 10000 hectáreas para la colonización italiana al oeste de Sabalito en la zona de Coto Brus, con la finalidad de: 1) favorecer en varias formas la inmigración y 2) construir y mantener una carretera de acceso a la colonia. Por su parte, la compañía italiana se obligaba a instalar entre 250 y 300 familias (de las cuales el 20\% debía ser costarricense), ayudar a los emigrados, distribuir la tierra, organizar la 
nueva comunidad en casas y escuelas, así como construir una central eléctrica, un aserradero y un beneficio (Sansonetti, Vito, 1995).

Las motivaciones de estos emigrantes italianos se fundamentaban, por una parte, en la difícil situación de postguerra que atravesaba Italia, en la cual los problemas sociales y económicos eran evidentes y por otro lado, en que Costa Rica —al igual que el resto de América Latina - presentaba condiciones idóneas para la inmigración. Luego de la Guerra Civil de 1948, existía un buen clima en la economía costarricense, así como, un ambiente general de bienestar creado por la reconstrucción europea después de la Segunda Guerra Mundial y el alza de los precios del café en el mercado internacional (Bariatti; 2001).

Según cuenta el comandante Sansonetti (fundador de San Vito), entre las figuras costarricenses que le han brindado consejo $y$ apoyo (Sansonetti, Vito, 1995) están su cuñado Luis Demetrio Tinoco Castro (abogado y político, Secretario de Educación Pública (1940-1944) en la administración del Dr. Calderón Guardia y Ministro de Relaciones Exteriores (1958-1961) en la de don Mario Echandi), Lino Vicarioli (agrónomo italiano, fungió como Director General del Ministerio de Agricultura), César Dondoli (geólogo italiano, fundador de la Geología en Costa Rica) y Gastón Bartorelli (ingeniero italiano, quien trabajó para el Ministerio de Obras Públicas). Este último, además:

...diseñó y dirigió una cantidad impresionante de edificaciones...[como]...el Quiosco del Parque Central, la Capilla del Asilo Chapuí, partes del Hospital San Juan de Dios, la vieja sede del Instituto Nacional de Seguros, la Nunciatura Apostólica, la Casa Italia, las facultades de Ingeniería Civil y de Agronomía de la Universidad de Costa Rica, el primer edificio del Banco Nacional, el Colegio Sagrado Corazón, el Mercado Central, Tienda La Gloria, la Farmacia Jara, la Tienda el Globo, el Edificio Musmanni, el Edificio Tienda Simón y el Edificio Tienda Regis y Miniatura (Bariatti. 2001: 278).
El hecho de que todas estas eminentes figuras profesionales que rodearon a Sansonetti y su proyecto de colonización podría interpretarse como un posible interés institucional costarricense por las prácticas eugenésicas.

La influencia occidental de la idea del progreso junto con las teorías científicas eugenésicas (darwinismo social) promovieron desde finales del siglo XIX hasta mediados del Siglo Xx en América Latina — por los propios Estadosla promulgación de proyectos de inmigración de trabajadores y colonizadores campesinos europeos hacia zonas de escasa población. Costa Rica no fue una excepción pues su población:

...aquella ubicada en el Valle Central se considera[ba] "racialmente" homogénea $y$ predominantemente blanca. Los sectores dominantes, aunque satisfechos con la composición étnica de la población, están para entonces convencidos de que su escaso número es impedimento para el progreso...

Los gobernantes ya desde mediados del siglo XIX decidieron apoyar empresas de colonización siempre y cuando, por supuesto, estas trajeran la ansiada población europea (Alvarenga, 2002).

En el caso específico de Costa Rica, las leyes como la Ley 1316 de 1951, relacionada con la aprobación del contrato suscrito entre el Gobierno de la República y la Sociedad Italiana de Colonización Agrícola de Roma, es un claro ejemplo de la promoción de una política eugenésica cuyo fin es atraer población europea blanca. Estas ideas eugenésicas de la "raza ideal" están expresadas en el discurso institucional costarricense y un ejemplo de ello es el discurso del ex ministro Volio Guardia en conmemoración de los 30 años de fundación de San Vito de Java.

\section{A PROPÓSITO DE LAS CUATRO NOCIONES FUNDAMENTALES DEL ANÁLISIS DEL DISCURSO EN VAN DIJK}

El discurso del ex ministro Volio Guardia — pronunciado el 28 de febrero de 1982, en 
conmemoración de los 30 años de San Vitopertenece al género formal del discurso, preparado como texto escrito $y$ luego leído ante un público. Además, aparece publicado en la página 7, en el prólogo del libro Quemé mis naves en las montañas, del fundador de la ciudad, Vito Sansonetti. Dicho discurso cumple con una de las tesis principales de van Dijk (2008), en el sentido de que los usuarios del lenguaje al utilizar texto o habla, llevan a cabo acciones de índole política o social. Volio Guardia en su discurso político refleja las políticas eugenésicas del discurso institucional costarricense. $\mathrm{Su}$ discurso constituye una práctica social puesto que comunica estereotipos étnicos y a la vez, contribuye a la reproducción del sistema social del racismo (Ibid). Volio argumenta a favor de la inmigración de los italianos a San Vito, quienes por ser europeos, contribuyen al desarrollo nacional. Su discurso, a la vez que refleja parte del debate eugenésico para la construcción de la identidad nacional, permite perpetuar el discurso institucional costarricense.

Por lo tanto, teniendo presente la idea de que los sujetos llevan a cabo acciones de índole política y social en el momento de argumentar o escribir, se analizará dicho discurso desde el contexto en que se dice, el momento de la historia y el objetivo del discurso. Todos estos elementos entran en juego simultáneo cuando el entonces Ministro se dirige a su público y cuando Sansonetti publica dicho discurso en el prólogo de su libro. Para analizar este discurso político y para establecer vínculos teóricos entre el discurso y la sociedad, son necesarias las nociones de "acción", "contexto", "poder" e "ideología".

\subsection{SOBRE LA ACCIÓN}

Para van Dijk, el discurso es:

...un fenómeno práctico, social y cultural... [pues]... los usuarios del lenguaje...realizan actos sociales y participan en la interacción social...[la cual]...está, a su vez, enclavada en diversos contextos sociales y culturales, o encuentros institucionales [verbigracia el discurso de Volio] (2008: 21).
Este enfoque como interacción social implica orden y organización (lo cual se refleja en dicho discurso analizado, pues las palabras y las oraciones siguen un orden y coherencia) que se traduce "...en secuencias de actos mutuamente relacionados" (ibid). El Estado costarricense desde los últimos decenios del siglo XIX hasta mediados del siglo xx, ha promovido de manera continua, proyectos de inmigración de trabajadores y colonizadores europeos, que contribuyeron en el proceso de la creación de la identidad nacional. Esta política eugenésica coincide con el resto de los países de latinoamérica, cuyos gobiernos incentivaron inmigraciones masivas desde Europa. En el caso de Costa Rica, con la convicción de que el escaso número de población homogénea y blanca sería un impedimento para el progreso, el Estado en 1850, crea la Junta Protectora de las Colonias con el fin de promover la inmigración de colonos europeos (Alvarenga, 2002). Muchos proyectos fracasaron pero el proyecto colonizador de San Vito de Java fue un éxito (Bariatti, 2011).

Al definir el discurso como acción, se le concibe como "...una compleja jerarquía de diferentes actos en distintos niveles de abstracción y generalidad, por lo cual hacemos $\mathrm{X}$ mediante o mientras hacemos Y" (van Dijk, 2008: 24). Así, en el discurso del ministro Volio, dichas jerarquías de la acción se reflejan en diferentes actos e intenciones: Volio Guardia al dar su discurso, al mismo tiempo representa al gobierno, hace un acto conmemorativo por los 30 años de la llegada de los italianos a San Vito, argumenta a favor de la influencia positiva de dicha inmigración en el quehacer nacional $y$ defiende la posición eugenésica institucional. A la colonización italiana, en las selvas de Coto Brus, Volio Guardia la describe como "provechosa y constructiva" pues sus actores son "intrépidos" y constituyen "sangre nueva".

\subsection{SOBRE EL CONTEXTO}

El discurso está situado en un contexto $y$ se encuentra connotado $y$ denotado por este. El discurso se realiza en una situación social, la cual influenciará la producción o interpretación de las estructuras del texto y el habla, ya sea a los actores, sus roles, sus propósitos, el tiempo 
$y$ el lugar en que se realiza (van Dijk, 2008). En el ejemplo del discurso de Volio, las categorías del tiempo y del lugar están constituidas por la fecha del 28 de febrero de 1982, día en que se conmemoran en un acto oficial, los 30 años del aniversario de la ciudad de San Vito, lugar donde se desarrolló exitosamente, el proyecto de colonización incentivado por las clases gobernantes.

Los participantes están constituidos por el Ex Ministro de Agricultura, quien da el discurso y la audiencia que lo escucha, los pobladores de San Vito y representantes institucionales que llegan a la conmemoración de los 30 años. Además, al encontrarse el discurso publicado trece años después en el libro del fundador de San Vito, Vito Sansonetti, la esfera de los receptores aumenta. Para comprender mejor dicho discurso político, hay que tomar en cuenta las propiedades relevantes de los participantes, las cuales se relacionan con el contexto $y$ por ende, con la estructura del discurso.

Siguiendo a van Dijk, el contexto es manifestado por el discurso al afirmar que "...los usuarios del lenguaje utilizan activamente los textos y el habla... como miembros de categorías sociales" (ibid: 22). En nuestro texto, se observa que Volio en su discurso, es simpatizante de las políticas eugenésicas del discurso institucional costarricense, al desempeñar simultáneamente los roles de Ministro de Agricultura, de representante del Gobierno de turno $y$ de miembro de un partido político (Partido Liberación Nacional), construyendo así su subjetividad. La posición del ministro como hablante viene del ámbito público, institucional $y$ formal.

\subsection{SOBRE EL PODER}

Es necesario comprender la naturaleza del poder social (van Dijk, 2008), definido como una relación específica entre grupos sociales o institucionales, donde un grupo tiene control sobre otro. El discurso no es únicamente un medio para la realización del poder sino también un recurso de poder. Siempre hay alguien que detenta dicho acceso. El control de las estructuras del discurso implica un control del contexto. Es decir, los receptores no tienen información alternativa: la credibilidad y retórica persuasiva del discurso público puede ser tal, que muchos terminan por adoptar las creencias expresadas por esos discursos.

A grandes rasgos, hay dos tipos de poder coercitivo (a través de la fuerza física) y mental (a través del control de las intenciones o propósitos de las personas). Este último es el poder que sale a relucir en el discurso de Volio: legitimado con la investidura de ministro, ejerce su poder a través de su discurso político. En el texto, Volio -como actor político- al narrar el origen de la ciudad de San Vito, utiliza una de las técnicas para legitimar su poder, la autopresentación positiva (op. cit.): "un buen día, se presentó a mi despacho del Ministerio de Agricultura e Industrias el Comandante Sansonetti para proponerme un proyecto de colonización agrícola con inmigrantes italianos. La idea me pareció muy provechosa..." (cfr. Sansonetti, Vito, 1995: 7). La otra cara de la moneda de esta técnica de legitimación es la deslegitimación, en lo cual los otros son presentados negativamente: "si repasamos la historia de las anteriores y diversas inmigraciones de agricultores a nuestro suelo, podemos darnos cuenta que todas fracasaron por diversas razones" (op. cit. $)^{2}$.

Hay dos nociones relacionadas con el poder: hegemonía y consenso. El término hegemonía se refiere al poder social: se "naturaliza" el consenso. La 'blanquitud' (Jiménez, 2009 y Sandoval, 2008) buscada por el discurso institucional costarricense, a fuerza de repetirla en diversos tipos de discurso, se vuelve consensuada, como algo natural. El discurso de Volio no es un hecho aislado, sino parte de una concatenación de textos y habla institucionales.

\subsection{SOBRE LA IDEOLOGÍA}

La ideología es otra de las nociones que establece un vínculo entre el discurso y la sociedad, cuya función es reproducir y legitimar el discurso de los grupos dominantes: "una de las estrategias para realizar esa legitimación [de un modo persuasivo] es, por ejemplo,

\footnotetext{
$2 \quad$ El énfasis en negrita fue hecho por el mismo Sansonetti, coincidiendo así con el discurso institucional.
} 
presentar la dominación como impuesta por Dios, natural, benigna, inevitable, o persuadir al grupo dominado para que simplemente dé por hecha esa relación social" (van Dijk, 2008: 51). En el texto de Volio, se encontró cómo sus palabras ayudan a reproducir el discurso institucional de los grupos de poder, al presentar como un hecho natural $y$ benigno el proyecto de colonización: "Costa Rica necesita capitalizar las experiencias positivas. Nuestro país se beneficiaría grandemente con otros "San Vito de Java" y otros Sansonetti. Ojalá lleguen gentes de esos merecimientos a nuestras costas y quemen allí sus naves" (Sansonetti, Vito, 1995: 7). Volio le dice a su audiencia y Sansonetti a sus lectores en su libro, cuál es la posición que deben asumir y que deben pensar acerca de dicha política eugenésica.

De este modo las ideologías sirven para coordinar las prácticas sociales dentro del grupo y regular su interacción social con los miembros de otros grupos.

Una vez compartidas, las ideologías aseguran que los miembros de un grupo actuarán en general de modos similares en situaciones similares, serán capaces de cooperar en tareas conjuntas, $y$ contribuirán así a la cohesión grupal, la solidaridad y la reproducción exitosa del grupo (van Dijk, 2008: 52).

Por ejemplo, la ideología detrás del discurso institucional sugiere a todos los costarricenses que sean empáticos con proyectos de colonización extranjera, específicamente europea.

Siguiendo el ejemplo del discurso político de Volio (op. cit), se observa cómo se manifiestan estas propiedades estructurales del discurso. Para ello se toman en cuenta: la selección del tema, la organización esquemática, la lexicalización que sugiere nuestras propiedades positivas y sus propiedades negativas, el estilo y los mecanismos retóricos, tales como: contrastes, metáforas, hipérboles y eufemismos. La selección del tema, es decir, la inmigración europea como algo positivo, es guiado por las frases "la idea me pareció muy provechosa", “... treinta años después, podemos decir que Costa Rica cuenta con otra Meseta Central en Coto
Brus”. Esta política eugenésica -en términos de esa ideología del blanqueamiento y del centro del país- es reforzada, de manera coherente, por la organización esquemática de todo el discurso: inicia con la historia del proyecto de colonización italiana, la justificación para ello, el énfasis en lo bueno de los inmigrantes italianos $y$ el fracaso de otras inmigraciones, la atenuación de lo negativo de los primeros y la invisibilización de lo positivo de otras inmigraciones. Finaliza el discurso con la admiración de Volio, envestido de autoridad estatal, hacia los italianos y los costarricenses que los acompañaron.

Para el análisis ideológico del discurso, van Dijk (ibid: 59-63) aplica el Método del Cuadrado Ideológico, donde la autopresentación es positiva en contraposición con la presentación negativa del otro. Esta polarización ideológica se expresa en el discurso de Volio a través de referencias negativas hacia otros proyectos de colonización agrícola que han fracasado frente a la exitosa empresa colonizadora de San Vito: "si repasamos la historia de las anteriores y diversas inmigraciones de agricultores a nuestros suelo, podemos darnos cuenta que todas fracasaron por diversas razones. La Colonia Italiana de San Vito de Java ha sido la excepción" (Sansonetti, Vito, 1995: 7). Se encuentran en contraposición, ejemplos de lexicalización positiva hacia los inmigrantes italianos de San Vito de Java en las siguientes frases: "empresas más provechosas y más constructivas", "intrépidos inmigrantes", "idea muy provechosa", "era conveniente traer sangre nueva a nuestro campo", "era útil recibir la cultura de un pueblo laborioso" y "un reto prometedor".

A su vez, Sansonetti desarrolla su autoesquema que lo define, a él $-y$ a su familia- como una persona con destrezas especiales para llevar a cabo la colonización agrícola en San Vito. Esta autorepresentación es sumamente positiva. En el resto del libro, narra su origen genealógico - el cual es una analogía a Cristóbal Colón, quien era navegante italiano-, que viene de un respetado almirante naval italiano, siendo él mismo, siguiendo los pasos de su padre, comandante naval y héroe durante la Segunda Guerra Mundial. Su matrimonio no fue con 
cualquier mujer, sino con una integrante de la oligarquía costarricense: "así, de un matrimonio de una dama costarricense con un marino italiano, nació una ciudad y se desarrolló una región" (Sansonetti, Vito, 1995:15). Volio en su discurso político defiende esta idea del mesías italiano: "bajo el comando de Vito Sansonetti", "sangre nueva", "ha estado por el frente la tenacidad, el espíritu de sacrificio y el amor a la tierra de la familia Sansonetti, hoy tan "tica" como somos todos nosotros" y "nuestro país se beneficiaría grandemente con otros Sansonetti" (ibid: 7).

\section{ALGUNAS CONCLUSIONES PRELIMINARES}

En el discurso de Volio, se observa cómo los principales conceptos trabajados: acción, contexto, poder e ideología, de Teun van Dijk (2008), sugieren la construcción de significados del discurso institucional costarricense y sus políticas eugenésicas.

De manera que la representación mental sea compartida por todos los miembros de la sociedad costarricense, dicho discurso institucional es compartido socioculturalmente $y$ aplicado por los miembros del grupo en sus prácticas cotidianas. Una de estas prácticas la constituyen los discursos políticos. Volio no escatima recursos en aprovechar su intervención en la celebración de los 30 años de San Vito de Java para reproducir el discurso institucional.

Sin desmerecer el sacrificio de los inmigrantes italianos en San Vito -quienes fueron llevados a una selva virgen en el Valle de Coto Brus sin caminos, puentes ni protección sanitaria y sin ningún conocimiento sobre cultivo tropicalmuchos de ellos llegaron allí y colonizaron grandes extensiones de tierra. Sin embargo, algunos no resistieron y se fueron después de vender sus terrenos para trasladarse a Australia, Estados Unidos u otros lugares. Los Sansonetti, por ejemplo, ya no viven en San Vito. Por supuesto, otros se quedaron, muchos se mezclaron con los ticos y amaron a su nuevo terruño.

\section{BIBLIOGRAFÍA}

Alvarenga Venutolo, Patricia. "La inmigración extranjera y la nación costarricense". ISTMO Revista Virtual de Estudios Literarios y Culturales Centroamericanos 4. Julio-diciembre 2002. En: <http://istmo.denison.edu/n04/ articulos/migrantes.html>.

Bariatti, Rita. Italianos en Costa Rica 1502-1952. De Cristóbal Colón a San Vito de Java. San José, Costa Rica: Universidad Autónoma de Centroamérica (UACA), 2001.

Bariatti, Rita. Italianos en América Central. De Cristóbal Colón a la segunda posguerra. San José, Costa Rica: Editorial Alma Mater, 2011.

Chilton, Paul y Schaffner, Christina. "Discurso y política". El discurso como interacción social: estudios sobre el discurso II. Barcelona, España: Editorial Gedisa, 2008.

Franceschi, Temistocle. Lingua e cultura di una comunità italiana in Costa Rica. Firenze, Italia: Valmartina Editore, 1970.

Hall, Carolyn. El café y el desarrollo históricogeográfico de Costa Rica. San José, Costa Rica: Editorial Costa Rica y Universidad Nacional, 1976.

Jiménez Matarrita, Alexander. La vida en otra parte. Migraciones y cambios culturales en Costa Rica. San José, Costa Rica: Editorial Arlekín, 2009.

Martinez, Róger. "San Vito: asentamiento italiano en Costa Rica". Herencia 10-11 (2-1). Editorial Universidad de Costa Rica, 1998-1999: 133-135.

República de Costa Rica. Colección de Leyes y Decretos. San José, Costa Rica: Imprenta Nacional, 1952.

San Vito De Java. "San Vito de Java 1952-1977: Bodas de plata". Memoria patrocinada por la comunidad sanviteña en sus 25 años de fundación. San Vito de Java, Costa Rica. 1977.

Sandoval G., Carlos. El mito roto. Inmigración y emigración en Costa Rica. San José, Costa Rica: Editorial Universidad de Costa Rica, 2008.

Sansonetti, Ugo. Coto Brus, là dove gli alberi sorreggevano il cielo. Mottola, Taranto, Italia: Scorpione Editrice, 2000. 
Sansonetti, Vito. Quemé mis naves en estas montañas: la colonización de la altiplanicie de Coto Brus y la fundación de San Vito de Java. San José, Costa Rica: Jiménez \& Tanzi Ltda., 1995.

Sansonetti, Vito. Cuentos de guerra a un país de paz. San José, Costa Rica: Litografía CODELCA, 1998.

Van Dijk, Teun A. Racismo y discurso de las élites. Barcelona, España: Editorial Gedisa, 2003.

Van Dijk, Teun A. "El discurso como interacción en la sociedad". El discurso como interacción social: estudios sobre el discurso II. Barcelona, España: Editorial Gedisa, 2008.
Van Dijk, Teun A. Discurso y contexto: un enfoque sociocognitivo. Barcelona, España: Editorial Gedisa, 2012.

Weizmann, H. G. Emigrantes a la conquista de la selva, estudio de un caso de colonización en Costa Rica: San Vito de Java. San Vito, Costa Rica: Comité de la Sociedad Cultural "Dante Alighieri" de San Vito, reimpreso con la autorización del Comité Intergubernamental para las Migraciones (сім) de Ginebra, Suiza. 1985.

Fecha de ingreso: $26 / 08 / 2013$ Fecha de aprobación: 13/11/2013 


\author{
$A N E X O^{3}$ \\ DISCURSO OFICIAL DEL EX MINISTRO DE AGRICULTURA \\ (1949-1953) ING. AGR. CLAUDIO ANTONIO VOLIO GUARDIA \\ EN OCASIÓN DE LOS 30 AÑOS DE SAN VITO DE JAVA
}

Pocas empresas ha acometido el país más provechosas y más constructivas: la colonización de las selvas de Coto Brus, por un grupo de intrépidos inmigrantes bajo el comando de Vito Sansonetti.

Un buen día, se presentó a mi despacho del Ministerio de Agricultura e Industrias el Comandante Sansonetti para proponerme un proyecto de colonización agrícola con inmigrantes italianos. La idea me pareció muy provechosa: había entonces grandes extensiones de tierra de vocación agrícola que no estaban integradas a la producción nacional; no había mayores presiones de nuestra población por más tierra; era conveniente traer "sangre nueva" a nuestro campo; era útil recibir la cultura de un pueblo laborioso que se identificara con el nuestro de inmediato; en fin, era un reto prometedor.

Este reto fue aceptado. Se ha trabajado con grandes dificultades; se ha tropezado con obstáculos de todo tipo. Sin embargo, treinta años después, podemos decir que Costa Rica cuenta con otra Meseta Central en Coto Brus.

Si repasamos la historia de las anteriores y diversas inmigraciones de agricultores a nuestro suelo, podemos darnos cuenta que todas fracasaron por diversas razones. La Colonia Italiana de San Vito de Java ha sido la excepción. Reconozco que varios factores lo han hecho posible pero, por sobre todo, ha estado por el frente la tenacidad, el espíritu de sacrificio y el amor a la tierra de la familia Sansonetti, hoy tan "tica" como somos todos nosotros.

Costa Rica necesita capitalizar las experiencias positivas. Nuestro país se beneficiaría grandemente con otros "San Vito de Java" y otros Sansonetti. Ojalá lleguen gentes de esos merecimientos a nuestras costas y quemen allí sus naves.

A los campesinos "ticos" que junto con los italianos domeñaron los bosques y han asentado allí sus familias, les rindo mi admiración, y mi agradecimiento como ciudadano porque ellos si han sabido hacer patria.

\section{Claudio Antonio Volio Guardia} 28 de febrero de 1982

Ing. Agr. Claudio Antonio Volio Guardia, Ministro de Agricultura en 1951, que negoció y firmó el contrato de colonización con el Comte. Vito Sansonetti, Agente General de la Sociedad Italiana de Colonización Agrícola de Roma, Italia. En ocasión de los 30 años de San Vito de Java escribió lo que antecede.

3 Copia textual de Sansonetti, Vito. Quemé mis naves en estas montañas: la colonización de la altiplanicie de Coto Brus y la fundación de San Vito de Java. San José, Costa Rica. Jiménez \& Tanzi Ltda., 1995: 7 
